\title{
Self/other Perception Mediates Between Personality and Suicidal Ideation in Young Adults
}

\author{
Emanuele Fino* \\ Paolo Iliceto** \\ Ugo Sabatello*** \\ Francesca Petrucci**** \\ Gabriella Candilera ${ }^{\star \star \star \star}$ \\ * Department of Developmental and Social \\ Psychology, Sapienza University of Rome \\ ** CEO of S\&P Statistics and \\ Psychometrics Ltd, Rome \\ *** Department of Pediatrics and Child \\ Neuropsychiatry, Sapienza University \\ of Rome
}

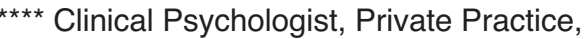
Rome

ITALY

\begin{abstract}
Background and Objectives: Individual differences in vulnerability to suicidal ideation have been recently addressed to internal working models of attachment. However, to date, research has mainly focused on adolescents. Few studies have investigated the role of adult attachment and personality dimensions in suicidal ideation, either examined from a mediational perspective. In this study, we aimed at testing a theoretical model in which Self/other perception mediates between personality and suicidal ideation in young adults.

Methods: Dimensions of Attachment (self/other perception), Personality (the Big Five factor model), and Suicidal Ideation (hopelessness and depression) were assessed in a community sample of 319 young adults from Northern Italy. Structural Equation Modeling and mediation analysis were conducted. Controlling for demographic variables (i.e., gender, age, education, and job), we tested three structural models.

Results: The final model confirmed our hypothesis that self/other perception mediates between personality (high neuroticism and low extraversion) and suicidal ideation, providing excellent fit to data.

Conclusions: In line with the conceptual framework of the Attachment Theory, findings suggest that failure to resolve attachment-related distress is related to the emergence of negative self/other models in adults. Such internal models are likely to attenuate the association between neuroticism and extraversion with depressive symptoms, hopelessness and suicidal ideation.
\end{abstract}




\section{Introduction}

Research investigating Suicidal Ideation (SI) in young adults has most consistently indicated depression and hopelessness as major predictors ${ }^{1-3}$. In particular, Beck ${ }^{4}$ conceptualized hopelessness as a core element of the "cognitive triad of depression". The Beck Hopelessness Scale $^{5}$ is the most generally employed measure of hopelessness. Research in both clinical and non-clinical populations ${ }^{6}$ supports a positive relationship between BHS scores and measures of depression, suicidal intent, and current SI.

Individual differences in vulnerability to depression, hopelessness, and SI have been recently addressed to internal working models of attachment ${ }^{7-9}$. The Attachment Theory ${ }^{10-13}$ posits that internal working models are mental representations shaped on early experiences with caregivers, guiding the individuals' thoughts, beliefs, and behaviors ${ }^{14}$. According to Bartholomew ${ }^{15}$, two dimensions of internal working models, i.e. the Self (perceived self-worthiness vs. reliance on other's judgment) and the Other (seek for closeness vs. avoidance), yield four prototypic patterns: Secure, preoccupied, fearful, and dismissing. Consistent with this theoretical framework, significant individual differences in the organization of stressful experience are expected throughout the lifespan. Previous studies reported that adults with less secure attachment styles are more likely to show less self-confidence and higher levels of negative affect ${ }^{16}$.

However, to date, research has mainly focused on the role of attachment in adolescents' psychopathology and suicidality ${ }^{17,18}$. Few studies have investigated the role of attachment styles in SI in adults. Recent investigations ${ }^{19}$ examined the relation between attachment styles and mental disorders in a national representative sample. The authors found that individuals with less secure at- tachment styles provided higher scores at measures of suicidal ideation, attempt, and mental disorders, supporting results from previous literature ${ }^{20}$. More recently, another study ${ }^{21}$ measured attachment security and social adjustment in relation to suicide attempt and major depressive episodes, finding that less secure attachment predicted increased risk of suicide attempt in depressed adults.

Interestingly, significant correlations between adult attachment dimensions and the Big Five factors of personality have been observed $^{22-24}$. In particular, previous studies showed that attachment security is negatively correlated with neuroticism, a personality trait that involves several negative emotions ${ }^{25}$, and positively correlated with extraversion, a trait associated with positive emotionality ${ }^{26}$. Nevertheless, to date, the specific role of adult working models of attachment and personality traits in hopelessness and SI has yet to be sufficiently clarified, either examined from a mediational perspective.

The purpose of the present study was to investigate the relations among dimensions of adult attachment, personality, and SI from a mediational perspective. All measured variables, as well as the underlying latent structural model, were tested in a sample of adults from the community. In particular, on the basis of previous literature, we hypothesized that young adults' internal working models, measured through self and other perception, mediate between the Big Five-neuroticism and -extraversion factors of personality and SI. Depressive symptoms and hopelessness were considered indicators of SI, as reported in previous research ${ }^{27,28}$. In order to determine the adequacy of the model fit to the empirical data, we postulated a specific relationship pattern a priori and then tested the hypothesis statistically. Finally, we tested mediation in order to identify a more comprehensive framework explaining direct and indirect effects among the three dimensions. 


\section{Method}

\section{Participants}

In the first half of 2013, 319 young adults, 173 males and 146 females participated to the study. Participants were from the local communities of two regions in the North of Italy, i.e. Lombardia and Veneto, either known to the authors or recruited via word of mouth. They participated on voluntary basis, and no economic compensation was provided. Informed consent was obtained, and the right to privacy was respected. Yet, all subjects were treated according to the guidelines of the World Health Organization and the declaration of Helsinki. Participants were aged 18 to 35 years, and no differences were found between the age of males (mean $=25.80 ; \mathrm{SD}=$ 3.74 ) and the age of females (mean $=26.22$; $\mathrm{SD}=4.06)\left(\mathrm{t}_{(317)}=0.95 ; \mathrm{p}=0.34\right)$. All participants were from middle class with various socio-economic backgrounds. No differences were found in the years of education $\left(\chi_{(2)}^{2}=\right.$ $3.56 ; \mathrm{p}=0.16)$ and in the working status $\left(\chi_{(1)}^{2}=0.19 ; \mathrm{p}=0.65\right)$. Socio-demographic characteristics are shown in Table 1.

Table 1

Socio-demographic characteristics of subjects

\begin{tabular}{llcccc} 
Characteristics & & Males $(\mathrm{N}=173)$ & Females $(\mathrm{N}=146)$ & Statistics & $\mathrm{p}$ \\
\hline Age (years) & & $25.80 \pm 3.74^{\mathrm{a}}$ & $26.22 \pm 4.06^{\mathrm{a}}$ & $\mathrm{t}_{(317)}=0.95$ & 0.34 \\
\hline Education (\%) & & & & $\chi_{(2)}^{2}=3.56$ & 0.16 \\
& $\leq 8$ & 12.1 & 19.2 & & \\
& $\leq 13$ & 50.9 & 43.2 & & \\
& $>13$ & 37.0 & 37.7 & & \\
\hline Job (\%) & & & & $\chi_{(1)}^{2}=0.19$ & 0.65 \\
& Unemployed & 53.2 & 50.7 & & \\
& Employed & 46.8 & 49.3 & & \\
\end{tabular}

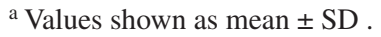

\section{Instruments}

Depressive symptoms were measured by the Beck Depression Inventory Second Edition (BDI-II: 29), a 21-item self-report. The internal consistency was assessed by means of Cronbach's Alpha (0.92). Respondents choose statements that reflect how they have felt over the past 2 weeks. BDI-II scores range between 0 and 63; categorical depression ratings are "minimal" (0-13), "mild" (14-19), "moderate" (20-28), and "severe" (29-63). The authors found, in their assessed clinical sample, a cut-off of 17 or greater with a $93 \%$ true-positive rate and a $18 \%$ false-positive rate.

Hopelessness was measured through the Beck Hopelessness Scale (BHS: 1, 5), a 20item true or false self-report scale developed to operationalize the construct of hopelessness. Responding to the 20 true or false items on the BHS, individuals can either endorse a pessimistic statement or deny an optimistic statement. The BHS may be used as a proxy indicator of suicide potential. Studies on the Italian population were carried out ${ }^{30}$ and led 
to successful validation of the scale $e^{31}$. In order to increase the response sensitivity, we replaced the original true/false response format with a five-point Likert-type one, including two extreme options of "Very Strongly Disagree" (zero) and "Very Strongly Agree" (four). The measure of Hopelessness was obtained through reversing the scoring of positive items, and then summing the 20 items to yield a total score ranging from 0 to 80 . In this sample the instrument showed a good reliability (Cronbach's Alpha $=0.83$ ).

The 9 Attachment Profile (9AP: Candilera, 2007) was used to measure attachment. The 9AP is a semi-projective test for assessing the quality of interpersonal relationships based on self/other perception and internal working models of adult attachment. Bowlby's ${ }^{13}$ notion of attachment representation involves ideas regarding both the self and others. A person's representation of the self and of others can be characterized by one of two orientations, i.e. positive or negative. This measure consists of seven basic pictures. Each picture represents a situation with one black figure and one or more white figures in different environments. The presentation of each picture is accompanied from two equal lists of nine differential semantics. On the first list, participants rate each of the differential semantics representing their self-perception on a 9-point scale. On the second list, participants' perception of the others is evaluated. The 9AP provides 18 bipolar scales regarding psychological and emotional constructs, 9 self-related and 9 other-related: Acceptance-rejection, friendliness-hostility, power-submission, security-insecurity, availability-unavailability, calm-agitation, satisfaction-dissatisfaction, independence-dependence, lack of competition-competition. Higher scores correspond to the first term of each bipolar scale (positive representation), lower scores to the second term (negative representation). In sum, we used the two general indicators, Self-perception (Cronbach's Alpha $=0.91)$ and Other-perception (Cronbach's Alpha $=0.89$ ), scored by adding up the respective scores on the nine self-related and the nine other-related scales, respectively.

The Zuckerman-Kuhlman-Aluja Personality Questionnaire (ZKA-PQ: 32) was used to assess personality. It is a 200 -item questionnaire based on the theoretical constructs of the alternative Five-factor model of personality. The instrument measures aggressiveness (physical aggression, verbal aggression, anger, and hostility), activity (work compulsion, general activity, restlessness, and work energy), extraversion (positive emotions, social warmth, exhibitionism, and sociability), neuroticism (anxiety, depression, dependency, and low self-esteem), and sensation seeking (thrill and adventure seeking, experience seeking, disinhibition, and boredom susceptibility/impulsivity). Adequate Alphas were reported for all factors (aggressiveness: 78 to .81 ; extraversion: .76 to .73 ; activity: .75 to .75 ; neuroticism: .74 to .79 ; sensation seeking: .70 to .72).

\section{Statistical Analyses}

Differences in socio-demographic characteristics were assessed through two-tailed ttests and Pearson's correlations for continuous variables, and chi-square tests with Yates's correction where appropriate for categorical variables.

Structural Equation Modeling (SEM) was employed. SEM relies on several statistical tests to determine the adequacy of the model fit to the empirical data. On the basis of the existing literature and consistently with the relevant theory, the researcher is asked to anticipate and test several relationship patterns. In SEM, it is possible to analyze relations between observed variables and latent vari- 
ables in addition to a measurement model. The measurement model specifies hypotheses about the relations between a set of observed variables and the unobserved variables or constructs that they were designed to measure. Yet, the structural equation model permits directional predictions among a set of independent or a set of dependent variables, and specifies the pattern of these relations regardless of whether they are observed or latent, allowing for a direct test of the theory of interest ${ }^{33}$.

We used the following criteria to evaluate the overall goodness-of-fit. The $\chi^{2}$ value close to zero indicates little difference between the expected and observed covariance matrices, with the probability level greater than 0.05 , evidencing the absence of meaningful unexplained variance. Moreover, to estimate a better goodness of fit, due to the fact that $\chi^{2}$ is sensitive to sample size, we calculated the ratio of $\chi^{2}$ to degrees of freedom. In addition to the $\chi^{2} / \mathrm{df}$ test, we utilized the Goodness-of-fit Index (GFI; 34), the Comparative Fit Index (CFI; 35), the Tucker-Lewis Index (TLI; 36), the Root Mean Square Error of Approximation (RMSEA; 37) and the Standardized Root Mean Square Residual (SRMR; 34). Indicators of a well-fitting model are evidenced by GFI, CFI and TLI greater than 0.95, RMSEA less than 0.06, and SRMR less than $0.08^{38-40}$.

We also tested mediation. Mediation is a process that links three variables, namely predictor, mediator, and outcome $\mathrm{e}^{41,42}$. In a structural model including multiple indicators for unobserved latent variables, and at least three (or more) latent variables, it is possible to assume a model with a latent predictor variable (A), a latent mediator variable (B), and a latent outcome variable $(\mathrm{C})$. In this case mediation refers to a framework explaining a more complex causal process among all three variables. In mediation, there is a $d i$ rect effect between an independent variable and a dependent variable, and there are indirect effects between an independent variable and a mediator variable, and between a mediator variable and a dependent variable. The mediation effect is the degree to which the direct effect changes when the researcher includes a mediator variable ${ }^{43}$.

We followed the recommendations provided by Baron and Kenny ${ }^{41}$, assuming four characteristics of mediation: The significant relation between $\mathrm{X}$ (predictor) and Y (outcome) (c), the relation between $\mathrm{X}$ (predictor) and $\mathrm{M}$ (mediator) $(a)$, the effect of the mediator on the outcome after controlling for the predictor $(b)$, and the remaining effect of $\mathrm{X}$ on $\mathrm{Y}$ after accounting for $\mathrm{M}\left(c^{\prime}\right)$. This effect is zero in full mediation, while in partial mediation the magnitude of the effect can vary. $c$ must be greater than $c^{\prime}$, and if $a$ and $b$ are significant and $c$ ' remains significant but $c$ ' $<$ $c$, a partial mediation process is supported ${ }^{44}$.

All analyses were carried out using SPSS 17.0 (SPSS Inc., Chicago, IL, USA). In the case of SEM, we used AMOS 16.0 (AMOS: Analysis of Moment Structures) and maximum likelihood estimation ${ }^{45}$.

\section{Results}

The descriptive statistics and zero-order correlations between the variables are presented in Table 2.

We first tested a model including all observed variables: Depression and hopelessness as indicators of Suicidal Ideation, self/ other perception as indicators of Attachment, and aggressiveness, activity, neuroticism, extraversion and sensation seeking as indicators of Personality. All fitted indices were statistically irrelevant $\left(\chi^{2}(24)=181.12(\mathrm{p}=0.000)\right.$; $\chi^{2} / \mathrm{df}=7.54 ; \mathrm{GFI}=0.89 ; \mathrm{TLI}=0.54 ; \mathrm{CFI}=$ 
Table 2

Descriptive Statistics and Zero-Order Correlations

\begin{tabular}{lrrrrrrrrrr} 
& Means & SD & 2 & 3 & 4 & 5 & 6 & 7 & 8 & 9 \\
\hline 1. Depression (BDI-II) & 6.07 & 4.23 & .49 & -.32 & -.31 & .04 & .03 & -.21 & .19 & -.05 \\
2. Hopelessness (BHS) & 17.02 & 4.09 & & -.33 & -.32 & .07 & .04 & -.25 & .24 & -.02 \\
3. Self perception (9AP) & 333.07 & 42.80 & & & .45 & .04 & .32 & .33 & -.13 & .24 \\
4. Other perception (9AP) & 319.55 & 43.66 & & & .07 & .31 & .30 & -.12 & .22 \\
5. Aggression (ZKA-PQ) & 99.13 & 20.31 & & & & .12 & .09 & .13 & .36 \\
6. Activity (ZKA-PQ) & 108.83 & 11.56 & & & & & .12 & -.06 & -.07 \\
7. Extraversion (ZKA-PQ) & 121.07 & 15.00 & & & & & & -.48 & .15 \\
8. Neuroticism (ZKA-PQ) & 91.40 & 19.43 & & & & & & & & .10 \\
9. Sensation seeking (ZKA-PQ) & 100.83 & 18.91 & & & & & & & \\
\hline
\end{tabular}

0.69; RMSEA = 0.14; SRMR = 0.10). Factor loadings were particularly low on aggressiveness (0.8), activity (.14), and sensation seeking (.15). The model was rejected.

Subsequently, we tested a second model in which aggressiveness, activity and sensation seeking were excluded. Nevertheless, we tested the relation between Personality and Suicidal Ideation (path $a$ ), under the condition that the path $b$ was constrained to zero, i.e. that path $b$ was not estimated. Also this model was not satisfactory, producing fit indices as follows: $\chi^{2}(7)=45.78(\mathrm{p}=<0.001)$; $\chi^{2} / \mathrm{df}=6.54 ; \mathrm{GFI}=0.95 ; \mathrm{TLI}=0.76 ; \mathrm{CFI}=$ $0.89 ;$ RMSEA $=0.13 ;$ SRMR $=0.07$. Both the paths were statistical significant, between Personality and Suicidal Ideation (-0.56), and between Personality and Attachment (0.63), as indicated in the measurement model by self/other perception.

Finally, in order to establish mediation, we tested a model in which path $b$ was estimated. This allowed us to test the mediation of Attachment between Personality and Suicidal Ideation. The fit of the model to the data was excellent $\left(\chi^{2}(6)=10.53(\mathrm{p}=0.08) ; \chi^{2} / \mathrm{df}\right.$ $=1.75 ; \mathrm{GFI}=0.98 ; \mathrm{TLI}=0.96 ; \mathrm{CFI}=0.98$;
RMSEA $=0.04 ;$ SRMR $=0.03$ ). The structural coefficient from Personality to Suicidal Ideation considerably decreased, toward almost zero, and it was statistical insignificant (-0.03), while the structural coefficient from Attachment to Suicidal Ideation increased $(-0.66)$, and all the fit indices improved significantly, providing evidence that the structures of the two models are different, and confirming our hypothesis.

The estimates of measurement model and the structural coefficients, in standardized metric for an easier interpretation, are depicted in Fig. 1. The loadings of indicator variables on the latent variables were all statistically significant.

\section{Discussion}

The data support a theoretical model in which self- and other-perceptions mediate between the Big Five-neuroticism and -extraversion traits and predictors of SI, i.e. depressive symptoms and hopelessness, in young adults from the community. We ob- 


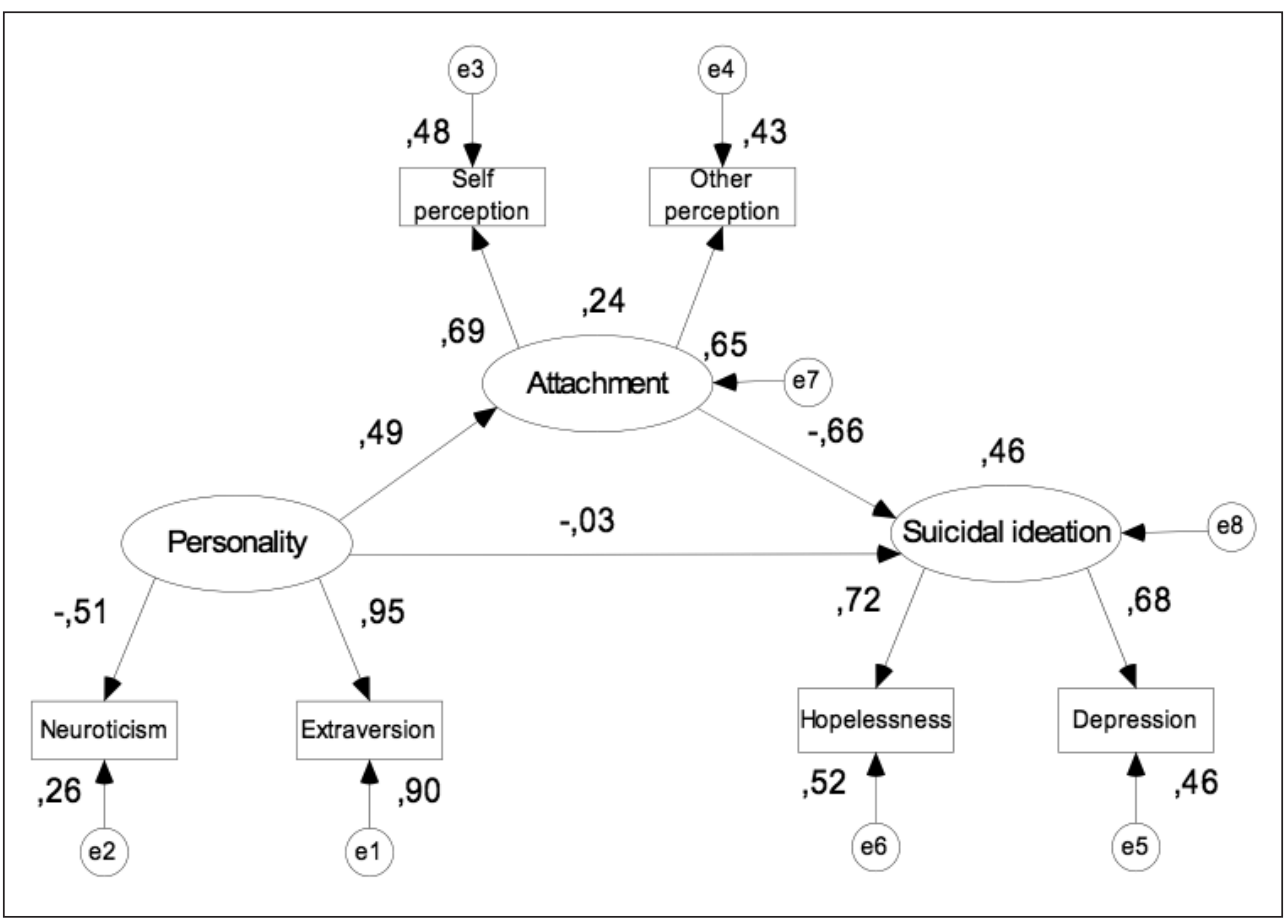

Figure 1. Mediation model.

served significant associations only when the mediator, i.e. Attachment, was entered into the model, attenuating the role of Personality.

These findings are in line with the conceptual framework of the Attachment Theory ${ }^{13}$, suggesting positive associations between individuals' attachment security and psychological functioning. Consistently, results from previous studies ${ }^{46-48}$ support the role of early life experiences in shaping the individuals' personality and psychological adjustment throughout the lifespan.

Accordingly, we found that young adults characterized by negative models of the self and the others are more likely to develop depressive symptoms and hopelessness, and to be more exposed to suicidal risk and ideation. One possible reason for such greater vulnerability may be a predisposition for these con- ditions in the face of stress, negative affect, and interpersonal difficulties, as evidenced elsewhere ${ }^{49-51}$. Yet, the overlap between suicidal behavior and insecure attachment could be interpreted as result of failure to resolve attachment-related distress, leading to the emergence of depressive symptoms, negative models of the self and the others, and lack of future expectations ${ }^{17}$.

Moreover, in the theoretical framework of the Attachment Theory, adult working models are conceived as stabilized cognitive constructs with regulatory function to personality and interpersonal behavior ${ }^{18}$. Accordingly, our model seems to suggest that individuals characterized by high neuroticism and low extraversion may be more likely to interpret experiences and behaviors on the basis of negative attachment patterns, with greater 
possibility of developing psychopathology and predisposing to suicidality ${ }^{19}$.

Interestingly, aggressiveness, activity and sensation seeking showed irrelevant loadings, and were excluded from the final model. These results are in line with previous literature $^{25}$ indicating on the one hand significant correlations between attachment security and extraversion, and attachment insecurity and neuroticism, and on the other hand minor associations with the other personality traits included in the Big Five factor model.

Nevertheless, our findings indicate a differentiation of personality and attachment dimensions in vulnerability to SI, in line with previous literature ${ }^{52}$. In fact, some suggested that an overlap between the two measures would be problematic, implying that attachment is redundant with major dimensions of personality $^{53}$, and research has consistently showed that "although there is some overlap or connection between attachment dimensions and the Big Five scales [...], the Big Five account for less than half of the variance in each attachment dimension", and "the two sets of measures are not redundant" 25 .

Although our findings cannot address specific implications for therapeutic interventions, they support the role of attachment as potential therapeutic target to prevent suicidal behavior in the context of depression ${ }^{21}$, especially in individuals with higher levels of hopelessness. An unresolved issue is the extent to which temperament, genetic, and other personality constructs may contribute to clarify the relations between the three dimensions investigated $^{18,23,53}$. Future research should implement more comprehensive methods and samples to test relations among these variables, in order to increase knowledge and provide guidelines for successful intervention.

The present study has some notable limitations. First, it should be noted that partici- pants were recruited through word of mouth, and selected on voluntary basis, within the same age range and resident in the same geographic area. A more heterogeneous sample selection would allow a better understanding of the role of social demographic characteristics and provide higher reliability. Second, we employed a community sample, and we consider critical for future research to test the mediational role of adult attachment between personality and predictors of SI on clinical samples. Third, although showing good psychometric properties, it is possible that the self-reported nature of data could have biased results by misleading or spurious correlations, and research integrating different instruments and procedures is needed. Finally, it is not possible to confirm the observed relations in terms of causal effects, given the cross-sectional nature of data. More research is needed to clarify the role of depressive feelings in affecting adult self/other perception, and possible repercussions to attachment-related stress.

\section{Acknowledgments}

This work was completed with the assistance of S\&P Statistics and Psychometrics Ltd, Rome, Italy.

\section{References}

1. Beck AT, Weissman A, Lester D, Trexler L. The measurement of pessimism: The Hopelessness Scale. J Consult Clin Psychol 1974; 42(6): 861-865.

2. Beck AT, Weismann A, Lester D, Trexler L. Classification of suicidal behaviours. Dimensions of suicidal intent. Arch Gen Psychiatry 1976; 33(7): 835-837.

3. Haaga DA, Yarmus M, Hubbard S, Brody C, Solomon A, Kirk L, et al. Mood dependency of self-rated attachment style. Cogn Ther Res 2002; 26(1): 57-71. 
4. Beck AT. Depression: Clinical, experimental, and theoretical aspects. New York: Harper Row; 1967.

5. Beck AT, Steer RA. Manual for the Beck Hopelessness Scale. San Antonio: The Psychological Corporation; 1989.

6. Kovacs M, Beck AT, Weissman A. Hopelessness: An indicator of suicidal risk. Suicide 1975; 5(2): 98-103.

7. Adam KS. Suicidal behaviour and attachment: A developmental model. In: Sperling MB, Berman WH, editors. Attachment in adults: Clinical and developmental perspectives. New York: Guilford; 1994. p. 275-298.

8. Davaji RBO, Valizadeh S, Nikamal M. The relationship between attachment styles and suicide ideation: the study of Turkmen students, Iran. Procedia Soc Behav Sci 2010; 5: 1190-1194.

9. Sheftall AH, Mathias CW, Furr RM, Dougherty DM. Adolescent attachment security, family functioning, and suicide attempts. Attach Hum Dev 2013;15(4):368-383.

10. Bartholomew K, Horowitz LM. Attachment styles among young adults: A test of a four-category model. J Pers Soc Psychol 1991; 61(2): 226-244.

11. Bowlby J. Attachment and loss: Vol. 1. Attachment. New York: Basic Books; 1969.

12. Bowlby J. Attachment and loss: Vol. 2. Separation. New York: Basic Books; 1973.

13. Bowlby J. Attachment and loss: Vol. 3. Loss, sadness and depression. New York: Basic Books; 1980.

14. Main M, Kaplan N, Cassidy J. Security in infancy, childhood and adulthood: A move to the level of representation. Child Dev 1985; 50(1-2): 821-827.

15. Bartholomew K. Avoidance of intimacy: An attachment perspective. J Pers Soc Psychol 1986; 51(6): 1173-1182.

16. Fraley RC, Shaver PR. Adult romantic attachment: Theoretical developments, emerging controversies, and unanswered questions. Rev Gen Psychol 2000; 4: 132-154.

17. Adam KS, Sheldon-Keller AE, West M. Attachment organization and history of suicidal behaviour in clinical adolescents. J Consult Clin Psychol 1996; 64(2): 264-272.

18. Mikulincer M, Shaver PR. Attachment in adulthood - Structure, dynamics, and change. New York: Guilford Press; 2007.

19. Palitsky D, Mota N, Afifi TO, Downs AC, Sareen J. The association between adult attachment style, mental disorders, and suicidality: Findings from a population-based study. J Nerv Ment Dis 2013; 201(7): 579-586.

20. Crowell JA, Treboux D. A review of adult attachment measures: Implications for theory and research. Soc Dev 1995; 4(3): 294-327.
21. Grunebaum MF, Galfalvy HG, Mortenson LY, Burke AK, Oquendo MA, Mann JJ. Attachment and social adjustment: relationships to suicide attempt and major depressive episode in a prospective study. J Affect Disord 2010; 123(1-3): 123-130.

22. Backstrom M, Holmes BM. Measuring adult attachment: A construct validation of two self-report instruments. Scand J Psychol 2001;42(1):79-86.

23. Fransson M, Granqvist P, Bohlin G, Hagekull B. Interlinkages between attachment and the Five-Factor Model of personality in middle childhood and young adulthood: A longitudinal approach. Attach Hum Dev 2013;15(2):219239.

24. Shaver PR, Brennan KA. Attachment style and the Big Five personality traits: Their connection with romantic relationship outcomes. Pers Soc Psychol B 1992; 18(5): 536-545.

25. Noftle EE, Shaver PR. Attachment dimensions and the big five personality traits: Associations and comparative ability to predict relationship quality. J Res Per 2006; 40: 179-208.

26. Costa PT, McCrae RR. Influence of Extraversion and Neuroticism on subjective well-being: Happy and unhappy people. J Pers Soc Psychol 1980; 38(4): 668-678.

27. Cheung YB, Law CK, Chan B, Liu KY, Yip PSF. Suicidal ideation and suicidal attempts in a population-based study of Chinese people: Risk attributable to hopelessness, depression, and social factors. J Affect Disord 2006; 90(23): 193-199.

28. Johnson J, Gooding PA, Wood AM, Taylor PJ, Pratt D, Tarrier N. Resilience to suicidal ideation in psychosis: Positive self-appraisals buffer the impact of hopelessness. Behav Res Ther 2010; 48(9): 883-889.

29. Beck AT, Steer RA, Brown GK. Manual for the Beck Depression Inventory-II. San Antonio: The Psychological Corporation; 1996.

30. Pompili M, Tatarelli R, Rogers JR, Lester D. The Hopelessness Scale: a factor analysis. Psychol Rep 2007; 100(2): 375-378.

31. Pompili M, Iliceto P, Lester D, Innamorati M, Girardi P, Tatarelli R. BHS. Beck Hopelessness Scale. Firenze: Organizzazioni Speciali; 2009.

32. Aluja A, Kuhlman M, Zuckerman M. Development of the Zuckerman-Kuhlman-Aluja Personality Questionnaire (ZKA-PQ): A Factor/Facet version of the ZuckermanKuhlman Personality Questionnaire (ZKPQ). J Pers Assess 2010; 92(5): 416-431.

33. Bollen KA. Structural equations with latent variables. New York: Wiley; 1989. 
34. Jöreskog KG, Sörbom DG. LISREL 8: User's Reference Guide. Chicago: Scientific Software International; 1996.

35. Bentler PM. Comparative fit indexes in structural models. Psychol Bull 1990; 107(2): 238-246.

36. Tucker LR, Lewis C. A reliability coefficient for maximum likelihood factor analysis. Psychometrika 1973; 38: $1-10$.

37. Steiger JH. Structural model evaluation and modification: An interval estimation approach. Multivar Behav Res 1990; 25(2): 173-180.

38. Browne MW, Cudeck R. Single sample cross-validation indices for covariance structure. Multiv Behav Res 1989; 24(4): 445-455.

39. Hu L, Bentler PM. Fit indices in covariance structure modeling: Sensitivity to underparameterized model misspecification. Psychol Methods 1998; 4(3): 424-453.

40. Hu L, Bentler PM. Cutoff criteria for fit indexes in covariance structure analysis: Conventional criteria versus new alternatives. Struct Equ Modeling 1999; 6(1): 1-55.

41. Baron RM, Kenny DA. The moderator-mediator variable distinction in social psychological research: Conceptual, strategic, and statistical considerations. J Pers Soc Psychol 2010; 51: 1173-1182.

42. MacKinnon DP, Fairchild AJ, Fritz MS. Mediation analysis. Annual Review of Annu Rev Psychol 2007; 58: 593-614.

43. Iacobucci D, Saldanha N, Deng X. A, meditation on mediation: Evidence that structural equations models perform better than regressions. J Consum Psychol 2007; 7(2): 139-153.

44. Hopwood CJ. Moderation and mediation in Structural Equation Modeling: Applications for early intervention research. J Ear Int 2007; 29(3): 262-272.

45. Arbuckle JL. Amos 16 User's Guide. Chicago: SPSS; 2007.
46. Cassidy J, Mohr JJ. Unsolvable fear, trauma, and psychopathology: Theory, research, and clinical considerations related to disorganized attachment across the life span. Clin Psychol Sci Pract 2006; 8(3): 275-298.

47. Sroufe LA. The role of infant-caregiver attachment in development. In: Belsky J, Nezworski T, editors. Clinical implications of attachment. Hillsdale: Erlbaum; 1988. p. 18-38.

48. Stepp SD, Morse JQ, Yaggi KE, Reynolds SK, Reed LI, Pilkonis PA. The role of attachment styles and interpersonal problems in suicide-related behaviours. Suicide Life Threat Behav 2008; 38(5): 592-607.

49. Balci V, Sevincok L. Suicidal ideation in patients with obsessive-compulsive disorder. Psychiatry Res 2010; 175(1-2): 104-108.

50. Brezo J, Paris J, Turecki G. Personality traits as correlates of suicidal ideation, suicide attempts, and suicide completions: A systematic review. Acta Psychiatr Scand 2006; 113(3): 180-206.

51. Mongrain M, Vettese LC, Shuster B, Kendal N. Perceptual biases, affect, and behavior in the relationships of dependents and self-critics. J Pers Soc Psychol 1998; 75(1): 230-241.

52. Fraley RC, Roisman GI, Booth-LaForce C, Owen MT, Holland AS. Interpersonal and genetic origins of adult attachment styles: A longitudinal study from infancy to early adulthood. J Pers Soc Psychol 2013; 104(5): 817-838.

53. Surcinelli P, Rossi N, Montebarocci O, Baldaro B. Adult attachment styles and psychological disease: Examining the mediating role of personality traits. J Psychol 2010; 144(6): 523-534.

Corresponding author:

Emanuele Fino

Department of Developmental and

Social Psychology at Sapienza

University of Rome

E-mail: emanuele.fino@uniroma1.it 